\title{
Lo esencial es invisible a los ojos (humanos): Patrones UV explican la mayor tasa de visita de los polinizadores a las flores amarillas del arbusto Cytisus scoparius
}

\author{
Varinia B. Vidal ${ }^{1} \&$ Alejandro G. Farji-Brener ${ }^{2,}$ 苂 \\ ${ }^{1}$ CRUB-Universidad Nacional del Comahue, San Carlos de Bariloche, Río Negro, Argentina. ${ }^{2}$ INIBIOMA, Laboratorio \\ Ecotono, Inibioma-CRUB, S. C. de Bariloche, Argentina.
}

\begin{abstract}
Resumen. A menudo, los polinizadores emplean los colores de las flores como señales indirectas del status de la recompensa. Sin embargo, los visitantes florales pueden percibir patrones del espectro cromático invisibles para el ojo humano. Cytisus scoparius (retama) es un arbusto abundante en bosques perturbados del NO de la Patagonia que presenta variación intra-específica en el color de sus flores. Nosotros evaluamos si la mayor tasa de visita de los polinizadores a las flores amarillas, previamente documentada, es consecuencia de un patrón UV más atractivo en este morfo. Contamos cada 4 días a lo largo de tres semanas el número de visitas a flores amarillas y rojas rociadas con bloqueador solar (bloqueando el espectro UV), flores sin bloqueador (control) y flores con bloqueador en su pedicelo (para controlar posibles efectos indeseados del bloqueador, como el olor). El efecto del bloqueador solar en los pétalos de las flores amarillas redujo la tasa de visita desde la primera medición. Al final del muestreo, las flores amarillas con bloqueador en sus pétalos fueron visitadas 7 veces menos que las flores control. Sin embargo, el efecto del bloqueador sobre los pétalos de las flores rojas recién fue evidente al final del muestreo, reduciendo la tasa de visita sólo 3 veces. Estos resultados sugieren que ambos morfos florales poseen patrones UV y que estos patrones atraen polinizadores, pero que su presencia estimula más visitas en las flores amarillas que en las rojas. Esto puede deberse a que el espectro UV es más contrastante en fondos amarillos que rojos. Finalmente, las flores amarillas y rojas con bloqueador solar en sus pétalos tuvieron una tasa de visita similar, sugiriendo que los polinizadores se ven más atraídos hacia las flores amarillas debido a la existencia de patrones UV y no por el color amarillo per se. Este trabajo resalta la importancia de analizar los patrones cromáticos invisibles al ojo humano para comprender mejor la relación entre las señales florales y las tasas de visita de los polinizadores.
\end{abstract}

[Palabras clave: color floral, interacciones planta-animal, Patagonia, polinización]

\begin{abstract}
Aвstract. The essential is invisible to the (human) eyes: UV patterns explain the increased visit rate of pollinators to the yellow flowers of the Cytisus scoparius bush. Flower colors are frequently used by pollinators as indirect signals of reward status. However, flower visitors are often UV-perceptive, a nonvisible chromatic spectrum for the human eye. Cytisus scoparius (scotch broom) is an abundant shrub of NW Patagonia that shows intra-specific variation in the color of their flowers. We evaluated whether the previously documented higher visitation rate of pollinators towards yellow flowers is consequence of a more attractive UV pattern in this floral morph. We counted the visitation rate every 4 days along 3 weeks in yellow and red flowers with sunscreens in their petals (which cancel the UV spectrum), control flowers (without sunscreen), and with sunscreen in their pedicels (to control undesirable sunscreen effects like odor). The addition of sunscreen in the petals reduced the visitation rate in the yellow floral morph since the first sampling day. At the last sampling day, yellow flowers with sunscreen in their petals were visited 7 times less than control ones. However, the effect of sunscreen in the petals of red flowers was only evident at the last sampling day, reducing the visitation rate at the end of sampling solely 3 times. These results suggest that both floral morphs show UV patterns and that these patterns attract pollinators, but also that UV patterns are particularly relevant for pollinators in yellow flowers. This may be an effect of a more contrasting UV pattern in yellow than in red background color. Finally, both flower color morphs with sunscreen in their petals showed similar visitation rate, suggesting that pollinators are attracted towards yellow flowers because of the existence of UV patterns rather than being attracted by the yellow color per se. This work emphasizes the importance of analyzing the chromatic patterns invisible to the human eye to better understand the relationship between flower signals and the visitation rate of pollinators.
\end{abstract}

[Keywords: flower color, Patagonia, plant-animal interactions, pollination]

Editor asociado: Diego Vázquez
Recibido: 27 de Marzo de 2019

Aceptado: 17 de Octubre de 2019 


\section{INTRODUCCIÓN}

Dado que la abundancia y calidad de las recompensas florales suelen ser indetectables de forma directa por los polinizadores, éstos seleccionan las flores que visitan en base a señales que indican de manera indirecta el status de la recompensa (Knauer and Schiestl 2014). Varios trabajos demostraron que ciertas características florales son indicadoras de la calidad de la recompensa y son utilizadas por los polinizadores para seleccionar las flores visitadas (i.e., "señales honestas"). Por ejemplo, en Raphanus sativus, los polinizadores son atraídos por flores con corolas más grandes, las cuales poseen más néctar y polen que las flores con corolas más pequeñas (Stanton and Preston 1988); y en Epilobium angustifolium visitan flores con mayor nivel de simetría, las que producen más néctar que flores asimétricas (Møller 1995). Dentro de las señales florales, el color es una de las más evidentes y ocurre en la gran mayoría de las flores (Papiorek et al. 2016). En consecuencia, el estudio de la relación entre los colores florales y sus visitantes es vital para comprender mejor la ecología y evolución de las interacciones entre las plantas con flores y sus polinizadores.

La variación intra-específica del color es una condición ideal para estudiar la relación entre las señales florales y la visita de polinizadores (Menzel and Shmida 1993). Primero, las variaciones en los colores son sencillas de detectar, pueden ser percibidas por los polinizadores y su importancia ha sido ampliamente documentada (Nuttman et al. 2006; Papiorek et al. 2013; Shrestha et al. 2016). Segundo, como esta variación en el color de las flores ocurre dentro de la misma especie, no es necesario emplear controles filogenéticos. Tercero, estos diferentes morfos florales pueden crecer muy cercanos entre sí y compartir las mismas condiciones ambientales y bióticas, lo cual facilita su comparación. Finalmente, los polinizadores pueden percibir cambios cromáticos intraespecíficos y responder modificando su tasa de visita (Nuttman et al. 2006).

Las variaciones en los colores florales pueden darse en longitudes de onda imperceptibles para el ojo humano, pero visibles para los polinizadores. Por ejemplo, las abejas pueden percibir la gama de colores que se extiende desde el ultravioleta (300 nanómetros) hasta el amarillo-anaranjado $(650 \mathrm{~nm})$, mostrando picos de sensibilidad para el ultravioleta, azul y verde (Primack 1982; Chittka 1996). Sin embargo, el ojo humano tiene un espectro de visión desde 400 a $750 \mathrm{~nm}$, con mayor sensibilidad para el azul, el verde y el rojo (Espinosa and Rivas 2011). Por lo tanto, los insectos polinizadores pueden percibir patrones en el espectro UV en flores que son completamente uniformes cromáticamente para el ojo humano (Koski and Ashman 2014). Se sabe que el 33\% de todas las especies de plantas presentan patrones ultravioletas en sus flores (Guldberg and Atsatt 1975). En muchas especies, estos patrones funcionan como "guías de néctar" ya que ayudan a los polinizadores a localizar las estructuras florales donde se produce el néctar y el polen, o simplemente enfatizan los contrastes (Jones and Buchmann 1974; Primack 1982). Por ende, es importante estudiar la existencia de patrones ultravioleta para comprender de manera cabal la relación entre estos patrones, la variación del color floral y la visita de los polinizadores.

Cytisus scoparius (L.) ("retama") es un arbusto muy abundante en bosques y matorrales con cierto grado de perturbación del NO de la Patagonia Argentina. Este arbusto representa un modelo de estudio ideal para analizar cómo variaciones intra-específicas del color en sus flores pueden interactuar con la existencia de patrones UV, afectando la tasa de visita de sus polinizadores. Por un lado, es un arbusto que presenta polimorfismo cromático en sus flores y abundancia diferencial; existen individuos con morfos florales amarillos (más abundantes) y con flores naranjas o rojas (menos abundantes) (Figura 1). Arbustos con diferentes morfos florales pueden encontrarse creciendo juntos en condiciones ambientales y de ensamble de polinizadores semejantes, lo que permite su comparación. Por otra parte, estudios previos demostraron que la mayor abundancia de arbustos con flores amarillas se debe a que sus polinizadores (mayoritariamente abejorros) eligen visitar flores de este color (Gavini and Farji-Brener 2015). Tanto en condiciones naturales como en experimentos en los que se manipuló el color, las flores amarillas fueron visitadas hasta 4 veces más que las flores de otros colores, efecto que fue independiente de la abundancia floral. En consecuencia, los arbustos con flores amarillas presentaron una producción de semilla/fruto $40 \%$ mayor que aquellos con flores rojas (Gavini and Farji-Brener 2015). Sin embargo, la atracción de los polinizadores por las flores amarillas podría deberse a la existencia diferencial de 

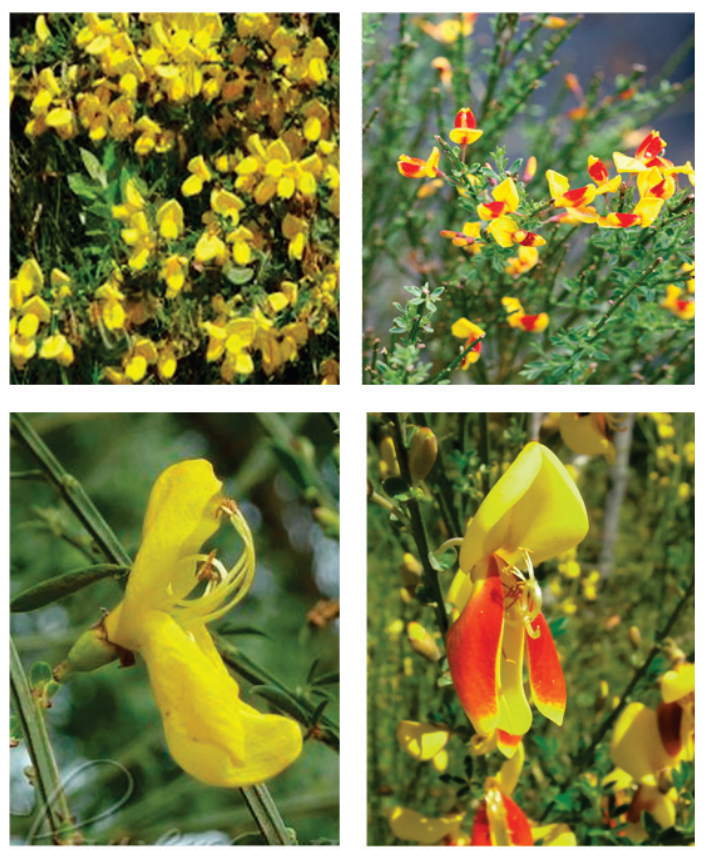

Figura 1. Arbustos de Cytisus scoparius con flores amarillas y rojas.

Figure 1. Shrubs of Cytisus scoparius with yellow and red flowers.

patrones UV en este morfo floral, aspecto hasta el momento desconocido. El objetivo de este trabajo fue determinar la existencia de patrones UV en los dos morfos florales de Cytisus scoparius (retama) y analizar su relación con las visitas florales. Para ello, en condiciones naturales monitoreamos las visitas de polinizadores a flores amarillas y rojas a las cuales modificamos sus patrones UV mediante el empleo de un protector solar y a sus respectivos controles. Planteamos la hipótesis de que la mayor tasa de visita a las flores amarillas que a las flores rojas, documentada previamente, se debe a la presencia de patrones UV más atractivos para los polinizadores en dicho morfo. Si esto es verdad, esperamos que a) las flores de ambos colores con su patrón UV modificado posean menos visitas que flores control, y $b$ ) la diferencia relativa en la tasa de visita entre flores con sus patrones UV modificados y su control sea mayor en las amarillas que en las rojas.

\section{MATERIAles y MÉTODOS}

\section{Sitio y especie de estudio}

Realizamos el muestreo durante noviembre de 2018 (primavera) alrededor de la ciudad de San Carlos de Bariloche, Argentina (41 $09^{\circ} \mathrm{S}$ $-71^{\circ} 18^{\prime} \mathrm{O}$ ), en sitios de bosque y matorral con gran abundancia de retama. La precipitación anual del área es $\sim 1600 \mathrm{~mm}$ y la temperatura media es $2^{\circ} \mathrm{C}$ en invierno y $13^{\circ} \mathrm{C}$ en verano. Trabajamos con la especie arbustiva exótica Cytisus scoparius (retama), originaria de Europa, que es muy abundante en el noroeste de la Patagonia. Sus flores papilionadas están compuestas por 5 pétalos. El pétalo superior se denomina estandarte, los dos laterales se llaman alas y los dos pétalos inferiores forman una quilla (Asturnatura 2008). En general, las flores son de color amarillo, aunque hay arbustos que presentan variantes florales con pétalos laterales de color rojo o naranja (Figura 1). Los polinizadores más comunes de la retama en el noroeste de la Patagonia son Apis mellifera (abeja doméstica) y Bombus spp. (abejorro) (Morales and Aizen 2002; Gavini and Farji-Brener 2015). Las flores sólo pueden ser abiertas si estos visitantes florales las estimulan, lo cual permite que el polen se libere de forma explosiva. De esta forma, la apertura de las flores es una señal inequívoca de que fue visitada por un polinizador (Gavini and Farji-Brener 2015).

\section{Metodología}

Para determinar la existencia de un patrón UV en las flores de la retama, su papel en la atracción de los polinizadores, y si el patrón UV difiere entre las flores rojas y amarillas, empleamos bloqueadores solares. Los bloqueadores solares dejan pasar las longitudes de onda superiores a $350 \mu \mathrm{m}$ (espectro visible al humano), pero absorben selectivamente las longitudes de onda entre

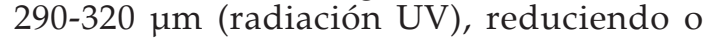
eliminando eventuales patrones cromáticos en dicho espectro. El empleo de bloqueadores solares para determinar la existencia de patrones UV en las flores y sus efectos sobre la tasa de visita fue probado previamente con éxito en otros trabajos, y es una herramienta simple, económica y accesible (Johnson and Andersson 2002; Koski and Ashman 2014). Trabajamos en 8 sitios donde estaban presentes simultáneamente arbustos de las dos variantes florales. En cada sitio se determinó un sector de $2 \mathrm{~m}$ lineales que incluía un arbusto con flores rojas y otro con flores amarillas de tamaño y cobertura semejantes; en cada planta se marcaron al azar 15 flores cerradas (i.e., no visitadas) por tratamiento. Los tratamientos fueron tres: 1) flores rociadas con bloqueador solar (Neutrogena $\circledR F P S 50$, spray), 2) flores sin bloqueador (control), 3) flores con bloqueador 
sólo en el pedicelo (control de eventuales efectos no deseados del bloqueador, como el olor). En consecuencia, monitoreamos un total de 720 flores, 360 de cada morfo floral (15 por 3 tratamientos, por 8 sitios). Para incrementar la probabilidad de visita, las flores fueron elegidas aleatoriamente en distintas posiciones de la periferia del arbusto, y cada una fue marcada en su pedicelo con alambres del mismo color, pero diferente patrón de nudo para cada tratamiento. Pese a que todos los arbustos elegidos fueron de tamaños similares, cuantificamos el diámetro de cada planta para determinar un eventual efecto de la cobertura sobre la tasa de visita.

Medimos el número de flores abiertas (i.e., visitadas) por tratamiento a lo largo del tiempo. Para eso, visitamos todos los arbustos muestreados a los 4, 8, 12 y 16 días de aplicar los tratamientos, y contamos cuántas de las flores marcadas por tratamiento pasaron de cerradas a abiertas (i.e., que fueron visitadas). En el día 8 se reaplicó protector solar en las flores cerradas para mantener su efectividad.

\section{Análisis de datos}

Se comparó el número de flores abiertas (variable respuesta) por tratamiento (i. e., con bloqueador solar, sin bloqueador solar y con bloqueador solar en el pedicelo) por cada morfo floral (amarillo y rojo), en los diferentes días de muestreo mediante un análisis de varianza de medidas repetidas. Tanto el tratamiento como el color fueron considerados factores fijos, el tiempo como la medida repetida, y el sitio que incluía a ambos arbustos como "bloque". Los diámetros de los arbustos fueron incluidos en el análisis como co-variable. Las comparaciones entre los diferentes tratamientos fueron analizadas a posteriori con una prueba de Tukey. Los datos se ajustaron a los supuestos del análisis, por lo que no fue necesario transformarlos.

\section{Resultados}

Las tasas de visita dependieron del efecto del bloqueador, el color, su interacción, el tiempo y el sitio. Por un lado, las flores amarillas recibieron más visitas que las rojas $\left(\mathrm{F}_{1,34}=18.9\right.$,

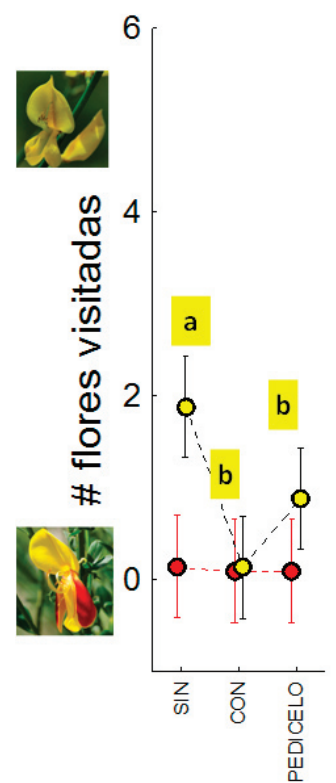

Dia 4

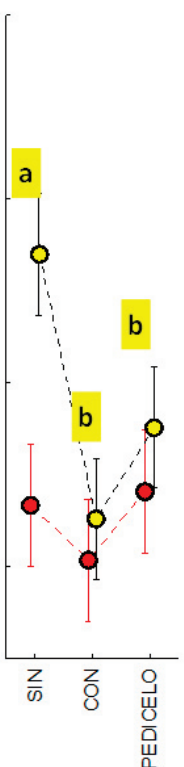

Dia 8

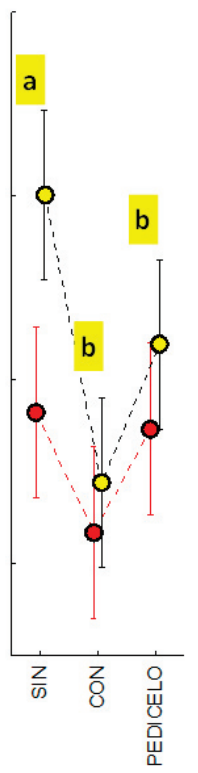

Dia 12

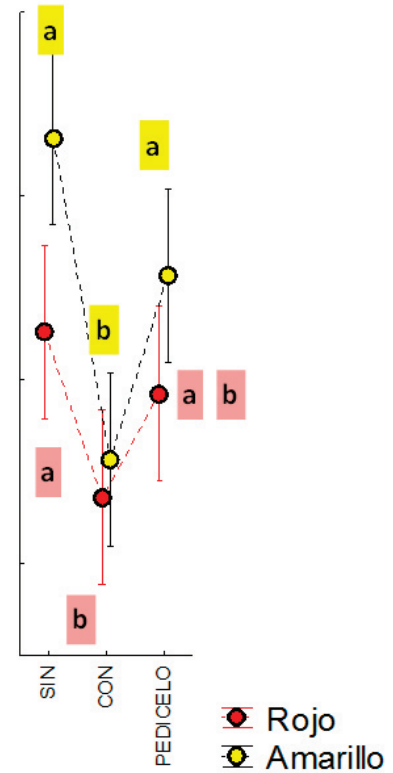

Dia 16

Figura 2. Número de flores visitadas de color amarillo y rojo con bloqueador solar en sus pétalos (CON), sin bloqueador solar (SIN, control), y con bloqueador solar en el pedicelo (PEDICELO, para controlar efectos indeseados del bloqueador, como el olor) a lo largo del muestreo. Distintos índices indican diferencias estadísticamente significativas, y su ausencia entre tratamientos del mismo morfo floral señala que no se detectaron diferencias estadísticamente significativas. Las barras representan intervalos de confianza del $95 \%$.

Figure 2. Number of yellow and red flowers visited with sunscreen in their petals (CON), without sunscreen (SIN, control), and with sunscreen in their pedicels (PEDICELO, to control undesirable sunscreen effects such as scent) along sampling. Different sub-indices mean statistical significant differences, and their absence among treatments of the same floral morph, the absence of statistical significant differences. Bars denote $95 \%$ confidence intervals. 
$P<0.001)$, y las flores sin tratar fueron más visitadas que las tratadas con bloqueador solar $\left(F_{2,34}=16.2, P<0.001\right)$. Sin embargo, los efectos de "tratamiento" y "color" interactuaron de manera significativa $\left(\mathrm{F}_{2,34}=4.2, P<0.02\right)$; las tasas de visita variaron entre los diferentes tratamientos, principalmente en las flores amarillas. Las flores sin bloqueador fueron las más visitadas, seguidas por aquellas con bloqueador en el pedicelo y, finalmente, las tratadas con bloqueador solar en sus pétalos (prueba de Tukey, $P<0.05$ ). Estas diferencias se fueron expresando más fuertemente a lo largo del tiempo (tratamiento $\mathrm{x}$ tiempo, $\left.\mathrm{F}_{6,102}=5.1, P<0.001\right)$. En el morfo de flores rojas sólo se observaron diferencias significativas entre tratamientos el último día de muestreo (día 16), momento en el que las flores rojas sin bloqueador presentaron más visitas que aquellas con bloqueador; las flores con bloqueador en el pedicelo presentaron un valor intermedio. Mientras tanto, en las flores amarillas, las visitas presentaron diferencias significativas entre tratamientos desde la primera fecha de muestreo (prueba de Tukey, $P<0.05)$, mostrando un claro gradiente de mayor a menor número de visitas en las flores sin bloqueador, con bloqueador en el pedicelo y con bloqueador en sus pétalos, respectivamente (Figura 2). En todas las fechas de muestreo las visitas a las flores amarillas sin bloqueador fueron significativamente mayores que a las flores amarillas con bloqueador solar en los pétalos. Sin embargo, sólo el último día de muestreo las visitas a las flores amarillas difirieron significativamente entre los tratamientos con bloqueador en los pétalos y con bloqueador sólo en el pedicelo, siendo significativamente mayores en este último. Finalmente, hubo sitios con mayor tasa de visita que otros $\left(\mathrm{F}_{734}=3.1, P<0.01\right)$, y el diámetro de las plantas no afectó la tasa de visita de los polinizadores a las flores $\left(\mathrm{F}_{1,34}=0.3\right.$, $P=0.58)$.

\section{DISCUSIÓN}

Este trabajo presenta tres resultados principales. Primero, confirma que los bloqueadores solares modifican los patrones UV en las flores. Segundo, revela la existencia de patrones UV en los morfos florales amarillos y rojos de Cytisus scoparius, lo que resalta su importancia en atraer visitantes florales. Y tercero, sugiere que la existencia del patrón UV en las flores amarillas es especialmente atractivo para los polinizadores, ofreciendo una explicación alternativa para la mayor tasa de visita que poseen las flores de este morfo (Gavini and Farji-Brener 2015). Estos resultados enfatizan la importancia de analizar la existencia de patrones UV en las variantes florales que difieren en el espectro visible, como una alternativa que pueda explicar la tasa de visita diferencial de los polinizadores hacia diferentes morfos florales.

Al final del período de muestreo, las flores con bloqueador solar en sus pétalos recibieron entre 1 y 3 veces menos visitas que las flores control. Esto sugiere que existen patrones UV en ambos morfos florales, que el bloqueador solar modifica estos patrones y que la presencia de estos patrones favorece la tasa de visita de los polinizadores. Pese a que las flores con bloqueador en el pedicelo acumularon visitas de manera más lenta que aquellas flores control, al final del muestreo, ambos tratamientos no difirieron en la tasa de visitas (Figura 2), lo cual indica que la menor tasa de visita a las flores con bloqueador solar se debe más a modificaciones en los patrones UV que a eventuales efectos indeseados, como su olor. Estos resultados no sólo evidencian la existencia de patrones UV en ambos morfos florales, sino que valida nuevamente el uso de bloqueadores solares para modificar patrones UV como una técnica económica, sencilla y accesible (Johnson and Andersson 2002; Koski and Ashman 2014).

Por otra parte, varios resultados sugieren que el patrón UV cumple un papel más importante en la atracción de los polinizadores en las flores amarillas que en las rojas. Primero, mientras que las flores amarillas control fueron más visitadas que aquellas con bloqueador solar en sus pétalos desde el primer día de muestreo (día 4), en el morfo rojo, este efecto recién fue evidente en el último período de muestreo (día 16). Segundo, mientras que el efecto del bloqueador solar en los pétalos de las flores amarillas hizo reducir la tasa de visita en promedio de 3.5 a 0.5 visitas (7 veces), en las flores rojas sólo lo redujo de 1.5 a 0.5 (3 veces). Estos resultados sugieren que el patrón UV del morfo amarillo es más atractivo para los polinizadores que los existentes en el morfo rojo. Finalmente, las flores amarillas y rojas con bloqueador solar en sus pétalos tuvieron similar tasa de visitas, sugiriendo que la variación en los patrones UV y no el color per se es el responsable de la atracción diferencial de los polinizadores hacia las flores amarillas. Sin embargo, los olores de las flores también podrían jugar un papel en la atracción de los visitantes florales. La 
tasa de visita similar entre morfos florales cuando el bloqueador solar fue colocado en el pedicelo (Figura 2), no permite descartar la idea alternativa de que de que el olor de la flor amarilla juegue un papel importante en la atracción de los visitantes florales, ya que el mismo podría verse enmascarado por la presencia de bloqueador. Futuros estudios deberán probar la existencia de compuestos odoríferos particulares en ambos morfos florales y su relevancia en la atracción de los polinizadores. Trabajos anteriores habían propuesto que la mayor tasa de visita hacia las retamas con flores amarillas se debía a una preferencia de los polinizadores hacia flores de este color (Gavini and Farji-Brener 2015). Este trabajo ofrece evidencias de la existencia de otro mecanismo: la existencia de patrones UV más atrayentes para los polinizadores en las flores amarillas que en las rojas. Es posible que, como en otras especies de plantas, las señales UV en fondo amarillo determinen patrones más contrastantes que en fondo rojo, color que es pobremente percibido por los insectos polinizadores (Primack 1982; Chittka 1996).

Se ha propuesto que el color es una atracción a distancia, mientras que los patrones de contrastes cromáticos orientan a los visitantes florales a las partes reproductivas de la flor (Lunau et al. 1996; Papiorek et al. 2016). Es común que flores completamente amarillas posean patrones UV que mejoren la atracción de las flores hacia sus polinizadores (Davies et al. 2012). Específicamente, diversos trabajos documentaron que las flores amarillas polinizadas por himenópteros presentan un patrón cromático donde el centro de la flor absorbe el espectro UV, mientras que su periferia lo refleja (Thompson et al. 1972; Harborne and Smith 1978; Koski and Ashman 2014). Este parece ser también el caso de la retama, al menos en su variante amarilla, en la que los pétalos que deben ser estimulados para acceder al polen son los que reflejan el espectro UV (ver http:/ / tiny.cc/v5mgkz). En resumen, nuestros resultados revelan que la mayor tasa de visitas a las flores amarillas que al resto de sus variantes cromáticas en retama no se debe a la atracción de los polinizadores por el color amarillo per se, sino a la existencia de patrones UV que posiblemente generan contrastes más atractivos u orientadores para los polinizadores en este morfo. Esto apoya la idea que flores con pétalos que presenten variación en la reflexión del espectro UV son más atractivas que aquellas uniformes en esa cualidad (Koski and Ashman 2014), enfatizando la importancia de analizar los patrones UV para comprender mejor la relación entre los colores florales, la tasa de visitas de los polinizadores y la abundancia diferencial de estas variantes cromáticas en la naturaleza.

Agradecimientos. Este trabajo es el producto final de la materia "Ecología de las Interacciones Planta-Animal" que se dicta en el Centro Regional Universitario Bariloche de la Universidad Nacional del Comahue para la Licenciatura y Doctorado en Biología. Versiones preliminares del proyecto fueron beneficiadas por discusiones entre compañeros de la cursada del año 2018. Los comentarios de dos revisores anónimos colaboraron a mejorar este trabajo.

\section{REFERENCIAS}

Asturnatura. Cytisus scoparius subsp. Scoparius (L.) Link. URL: www.asturnatura.com/especie/cytisus-scoparius-subspscoparius.htm (consultado el: 14/11/18). Núm. 181, 25/05/08. ISSN 1887-5068.

Chittka, L. 1996. Optimal sets of color receptors and color opponent systems for coding of natural objects in insect vision. J Theoret Biol 181:179-196. https:/ / doi.org/10.1006/jtbi.1996.0124.

Gavini, S. S., and A. G. Farji-Brener. 2015. La importancia del color: morfos florales, tasas de visita y éxito reproductivo en el arbusto Sarothamnus scoparius. Ecología Austral 25:204-211.

Guldberg, L. D., and P. R. Atsatt. 1975. Frequency of reflection and absorption of ultraviolet light in flowering plants. A Mid Nat 93(1):35-43. https://doi.org/10.2307/2424103.

Harborne, J. B., and D. M. Smith. 1978. Anthochlors and other flavonoids as honey guides in the Compositae. Bioch Syst and Ecol 6:287-291. https://doi.org/10.1016/0305-1978(78)90047-9.

Johnson, S. D., and S. Andersson. 2002. A simple field method for manipulating ultraviolet reflectance of flowers. Canadian J Botany 80:1325-1328. https://doi.org/10.1139/b02-116.

Jones, C. E., and S. L. Buchmann. 1974. Ultraviolet floral patterns as functional orientation cues in hymenopterous pollination systems. Anim Behav 22:481-485. https://doi.org/10.1016/S0003-3472(74)80047-3.

Knauer A. C., and F. P. Schiestl. 2014. Bees use honest floral signals as indicators of reward when visiting flowers. Ecol Letters 18:135-43. https://doi.org/10.1111/ele.12386.

Koski, M. H., and T. L. Ashman. 2014. Dissecting pollinator responses to a ubiquitous ultraviolet floral pattern in the wild. Funct Ecol 28:868-877. https://doi.org/10.1111/1365-2435.12242. 
Lunau, K., S. Wacht, and L. Chittka. 1996. Colour choices of naive bumble bees and their implications for color perception. J Comp Physiol A 178:477-489. https://doi.org/10.1007/BF00190178.

Menzel, R., and A. Shmida. 1993. The ecology of flower colours and the natural colour vision of insect pollinators: The Israeli flora as a study case. Biol Rev 68:81-120. https:/ /doi.org/10.1111/j.1469-185X.1993.tb00732.x.

Møller, A. P. 1995. Bumblebee preference for symmetrical flowers. Proc Natl Acad Sci 92:2288-2292. https://doi.org/ 10.1073/pnas.92.6.2288.

Morales, C. L., and M. A. Aizen. 2002. Does invasion of exotic plants promote invasion of exotic flower visitors? A case study from the temperate forests of the southern Andes. Biological Invasions 4:87-100. https:/ /doi.org/10.1023/A: 1020513012689.

Nuttman, C. V., F. M. Semida, S. Zalat, and P. G. Willmer. 2006. Visual cues and foraging choices: bee visits to floral color phases in Alkanna orientalis (Boraginaceae). Biol J Linnean Soc 87:427-35. https://doi.org/10.1111/j.10958312.2006.00582.x.

Papiorek, S., R. Junker, I. Alves'dos'Santos, G. Melo, L. Amaral'Neto, M. Sazima, and K. Lunau. 2016. Bees, birds and yellow flowers: pollinator'dependent convergent evolution of UV patterns. Plant Biol 18:46-55. https://doi.org/ $10.1111 /$ plb.12322.

Papiorek, S., K. Rohde, and K. Lunau. 2013. Bees' subtle color preferences: how bees respond to small changes in pigment concentration. Naturwissenschaften 100:633-643. https://doi.org/10.1007/s00114-013-1060-3.

Primack, R. B. 1982. Ultraviolet patterns in flowers, or flowers as viewed by insects. Arnoldia 42:139-146.

Shrestha, M., K. Lunau, A. Dorin, B. Schulze, M. Bischoff, M. Burd, and A. G. Dyer. 2016. Floral colors in a world without birds and bees: the plants of Macquarie Island. Plant Biol 18:842-850. https://doi.org/10.1111/plb.12456.

Stanton M. L. and R. E. Preston. 1988. Ecological consequences and phenotypic correlates of petal size variation in wild radish, Raphanus satious (Brassicaceae). Am J Bot 75:528-39. https://doi.org/10.1002/j.1537-2197.1988.tb13471.x.

Thompson, W. R., J. Meinwald, D. Aneshansley, and T. Eisner. 1972. Flavonols: pigments responsible for ultraviolet absorption in nectar guide of flower. Science 177:528-530. https://doi.org/10.1126/science.177.4048.528. 